\title{
A new model of economic order quantity associated with a generalized conformable differential-difference operator
}

\author{
Rabha W. Ibrahim,2, Rafida M. Elobaid ${ }^{3 *}$ (D) and Suzan J. Obaiys ${ }^{4}$
}

\section{"Correspondence:}

relobaid2000@yahoo.com

${ }^{3}$ Deanship of Educational Services,

Department of General Sciences,

Prince Sultan University, Riyadh,

Saudi Arabia

Full list of author information is

available at the end of the article

\begin{abstract}
The economic order quantity (EOQ) is the order quantity that minimizes the total holding costs and ordering costs. In this effort, we propose a design for EOQ by employing a conformable differential-difference operator, which accepts to minimize the EOQ.

Keywords: Conformable fractional calculus; Fractional operator; Economic order quantity; Cost function
\end{abstract}

\section{Introduction}

The economic order quantity (EOQ) was introduced by Harris [1] and modified by Woolsey [2] and Selen and Wood [3]. Ibrahim and Hadid [4] involved the differential term in its formula. Jaber et al. [5] presented a structure based on a physical scheme called entropy. Many studies have indicated new mathematical modeling of EOQ (see [6-9]).

Ibrahim et al. [10] suggested a model for EOQ by using a fractal idea (local fractional calculus) with cost functions corresponding to time at a returning time. They used the formula of Tsallis fractal entropy. The study presented an optimization relaxation problem for scheming a limited interval covering the optimal series distance. In addition, they introduced developments to calculate the optimal measure and the optimal order phase. The estimated EOQ by using a fractal definition did not recognize the error in the EOQ model. Therefore, one can think about a fractional calculus with a controller.

Anderson and Ulness introduced a new type of conformable calculus (CC) [11] involving a control term. Moreover, Ibrahim and Jahangiri [12] extended CC to the complex plane to study the analytic solution of a class of complex differential equations. Based on $\mathrm{CC}$, we generalize a differential-difference operator, type Dunkl of the first rank (CDD). Consequently, we employ this CDD to generalize the univex function, which we will utilize to minimize the EOQ system.

\section{Preparing}

In this section, we deal with the definitions of the important concepts of this effort.

(c) The Author(s) 2020. This article is licensed under a Creative Commons Attribution 4.0 International License, which permits use sharing, adaptation, distribution and reproduction in any medium or format, as long as you give appropriate credit to the original author(s) and the source, provide a link to the Creative Commons licence, and indicate if changes were made. The images or other third party material in this article are included in the article's Creative Commons licence, unless indicated otherwise in a credit line to the material. If material is not included in the article's Creative Commons licence and your intended use is not permitted by statutory regulation or exceeds the permitted use, you will need to obtain permission directly from the copyright holder. To view a copy of this licence, visit http://creativecommons.org/licenses/by/4.0/. 


\subsection{Conformable differential operator}

In this place, we introduce the most resent definition of the conformable differential operator (CDO), which can be located in [11].

Definition 1 (Conformable differential operator) A differential operator $\mathcal{D}^{\beta}, \beta \in[0,1]$ is conformable if and only if $\mathcal{D}^{0}$ is the identity operator and $\mathcal{D}^{1}$ is the ordinary differential operator. Particularly, the operator is conformable if and only if a differential function $\chi(t)$ satisfies

$$
\mathcal{D}^{0} \chi(t)=\chi(t) \quad \text { and } \quad \mathcal{D}^{1} \chi(t)=\frac{d}{d t} \chi(t)=\chi^{\prime}(t) .
$$

Moreover, in the theory of control systems, a proportional-differential controller for controlling resultant $v$ at time $t$ with two tuning criteria has the setting

$$
v(t)=v_{p} \Sigma(t)+v_{d} \frac{d}{d t} \Sigma(t)
$$

where $v_{p}$ is the proportional gain, $v_{d}$ is the derivative gain, and $\Sigma$ is the error between the formal variable and the actual variable. Based on (1), Anderson and Ulness [11] presented the common idea of CDO.

Definition 2 (A special class of conformable differential operators) For two continuous functions $v_{0}, v_{1}:[0,1] \times \mathbb{R} \rightarrow(0, \infty)$, we attain

$$
\mathcal{D}^{\beta} \chi(t)=v_{1}(\beta, t) \chi(t)+v_{0}(\beta, t) \chi^{\prime}(t)
$$

such that

$$
\lim _{\beta \rightarrow 0} v_{1}(\beta, t)=1, \quad \lim _{\beta \rightarrow 1} v_{1}(\beta, t)=0, \quad v_{1}(\beta, t) \neq 0, \forall t, \beta \in(0,1),
$$

and

$$
\lim _{\beta \rightarrow 0} v_{0}(\beta, t)=0, \quad \lim _{\beta \rightarrow 1} v_{0}(\beta, t)=1, \quad v_{0}(\beta, t) \neq 0, \forall t, \beta \in(0,1) .
$$

Definition 3 The integral operator corresponding to $\mathcal{D}^{\beta}$ is given by the following equality:

$$
\int \mathcal{D}^{\beta} \chi(t) d_{\beta} t=\chi(t)+\wp e_{0}\left(t, t_{0}\right)
$$

where $\wp \in \mathbb{R}, d_{\beta} t=\frac{d t}{v_{0}(t)}, v \neq 0$, and

$$
e_{0}(t, \kappa)=\exp \left(-\int_{\kappa}^{t} \frac{v_{1}(\beta, \varsigma)}{\left.v_{0}(\beta, \varsigma)\right)} d \varsigma\right)
$$

In our investigation, we request one of the following formulas of $v_{1}$ and $v_{0}$ :

$$
v_{1}(\beta, t)=(1-\beta) t^{\beta}, \quad \nu_{0}(\beta, t)=\beta t^{1-\beta}, \quad t \in(0, \infty),
$$




$$
\begin{aligned}
& v_{1}(\beta, t)=(1-\beta)|t|^{\beta}, \quad v_{0}(\beta, t)=\beta|t|^{1-\beta}, \\
& v_{1}(\beta, t)=\cos \left(\frac{\beta \pi}{2}\right) t^{\beta}, \quad v_{0}(\beta, t)=\sin \frac{\beta \pi}{2} t^{1-\beta}, \quad t \in(0, \infty),
\end{aligned}
$$

or

$$
v_{1}(\beta, t)=\cos \left(\frac{\beta \pi}{2}\right)|t|^{\beta}, \quad v_{0}(\beta, t)=\sin \frac{\beta \pi}{2}|t|^{1-\beta}, \quad t \in \mathbb{R} \backslash\{0\} .
$$

Finally, the conformable inner product between two continuous functions $\chi$ and $\varphi$ is given in the following formula:

$$
\langle\chi, \varphi\rangle=\int_{a}^{b} \chi(t) \varphi(t) e_{0}(b, t) d_{\beta} t
$$

\subsection{Differential-difference Dunkl operator}

Dunkl operator is an assembly of differential-difference operators taking the formula of first rank (see $[13,14])$

$$
\Delta_{\kappa} \chi(t)=\chi^{\prime}(t)+\kappa\left(\frac{\chi(t)-\chi(-t)}{t}\right), \quad \kappa \geq 0
$$

It extended a set of special functions and integral transforms in numerous variables linked with reflection groups. This type of operators has established many other operators. It is employed in the analysis of quantum body schemes. Lately, this operator is formulated in terms of fractional calculus and fractal (see $[15,16])$. In this place, we aim to introduce the conformable Dunkl operator (CDD) by using (2). Therefore, we have the following operator:

$$
\begin{aligned}
\Delta_{\kappa}^{\beta} \chi(t) & =\mathcal{D}^{\beta} \chi(t)+\kappa\left(\frac{\chi(t)-\chi(-t)}{t}\right) \\
& =v_{1}(\beta, t) \chi(t)+v_{0}(\beta, t) \chi^{\prime}(t)+\kappa\left(\frac{\chi(t)-\chi(-t)}{t}\right), \quad \kappa \geq 0 .
\end{aligned}
$$

It is clear that when $\beta \rightarrow 1$, Eqs. (10) and (2) coincide. Moreover, the CDD has the following properties.

Proposition 2.1 Let the CDD be given as in (10), where $\beta \in[0,1]$. Then, for constants $a$, $b, c$ and differential functions $\chi$ and $\varphi$, we have:

(i) $\Delta_{\kappa}^{\beta}[a \chi+b \varphi](t)=a \Delta_{\kappa}^{\beta}[\chi(t)]+b \Delta_{\kappa}^{\beta}[\varphi(t)]$;

(ii) $\Delta_{\kappa}^{\beta} c=c v_{1}(\beta, \cdot)$;

(iii) $\Delta_{\kappa}^{\beta}[\chi \varphi](t)=\chi(t) \Delta_{\kappa}^{\beta} \varphi(t)+\varphi(t) \Delta_{\kappa}^{\beta} \chi(t)-\chi(t) \varphi(t) v_{1}(\beta, t)+\kappa\left(\frac{\Phi(t)-\Phi(-t)}{t}\right)$, where

$$
\Phi(t):=\chi\left(\frac{\varphi}{2}-1\right)+\varphi\left(\frac{\chi}{2}-1\right)
$$

(iv) $\Delta_{\kappa}^{\beta}\left[\frac{\chi}{\varphi}\right](t)=\frac{\varphi(t) \Delta_{\kappa}^{\beta} \chi(t)-\chi(t) \Delta_{\kappa}^{\beta} \varphi(t)}{\varphi^{2}(t)}+\frac{\chi(t)}{\varphi(t)} v_{1}(\beta, t)+K_{\kappa}(t), \varphi \neq 0$, where

$$
K_{\kappa}(t):=\kappa\left(\frac{\left[\frac{\chi}{\varphi}\right](t)-\left[\frac{\chi}{\varphi}\right](-t)}{t}\right)-\frac{\kappa}{\varphi^{2}(t)}\left(\frac{\chi(t)-\chi(-t)}{t}\right)-\frac{\kappa}{\varphi^{2}(t)}\left(\frac{\varphi(t)-\varphi(-t)}{t}\right)
$$


Proof By using the definition of $\Delta_{\kappa}^{\beta}$, we have

$$
\begin{aligned}
\Delta_{\kappa}^{\beta} & {[a \chi+b \varphi](t) } \\
= & \mathcal{D}^{\beta}[a \chi+b \varphi](t)+\kappa\left(\frac{[a \chi+b \varphi](t)-[a \chi+b \varphi](-t)}{t}\right) \\
= & v_{1}(\beta, t)[a \chi+b \varphi](t)+v_{0}(\beta, t)[a \chi+b \varphi]^{\prime}(t)+\kappa\left(\frac{[a \chi+b \varphi](t)-[a \chi+b \varphi](-t)}{t}\right) \\
= & a\left[v_{1}(\beta, t) \chi(t)+v_{0}(\beta, t) \chi^{\prime}(t)+\kappa\left(\frac{\chi(t)-\chi(-t)}{t}\right)\right] \\
& +b\left[v_{1}(\beta, t) \varphi(t)+v_{0}(\beta, t) \varphi^{\prime}(t)+\kappa\left(\frac{\varphi(t)-\varphi(-t)}{t}\right)\right] \\
= & a \Delta_{\kappa}^{\beta} \chi(t)+b \Delta_{\kappa}^{\beta} \varphi(t) .
\end{aligned}
$$

This completes the first part. For the second part, a calculation implies that

$$
\Delta_{\kappa}^{\beta} c=\mathcal{D}^{\beta} c=v_{1}(\beta, t) c
$$

Now, we proceed to proving the multiplication rule under $\Delta_{\kappa}^{\beta}$. We have

$$
\begin{aligned}
& \Delta_{\kappa}^{\beta}[\chi \varphi](t) \\
&=v_{1}(\beta, t)[\chi \varphi](t)+v_{0}(\beta, t)\left[\chi \varphi^{\prime}+\varphi \chi^{\prime}\right](t)+\kappa\left(\frac{[\chi \varphi](t)-[\chi \varphi](-t)}{t}\right) \\
&=\chi(t)\left(v_{1}(\beta, t) \varphi(t)+v_{0}(\beta, t) \varphi^{\prime}(t)+\kappa\left(\frac{\varphi(t)-\varphi(-t)}{t}\right)\right) \\
& \quad+\varphi(t)\left(v_{1}(\beta, t) \chi(t)+v_{0}(\beta, t) \chi^{\prime}(t)+\kappa\left(\frac{\chi(t)-\chi(-t)}{t}\right)\right)-v_{1}(\beta, t) \chi(t) \varphi(t) \\
& \quad+\kappa\left(\frac{\left[\chi\left(\frac{\varphi}{2}-1\right)+\varphi\left(\frac{\chi}{2}-1\right)\right](t)-\left[\chi\left(\frac{\varphi}{2}-1\right)+\varphi\left(\frac{\chi}{2}-1\right)\right](-t)}{t}\right) \\
&:= \chi(t) \Delta_{\kappa}^{\beta} \varphi(t)+\varphi(t) \Delta_{\kappa}^{\beta} \chi(t)-v_{1}(\beta, t) \chi(t) \varphi(t)+\kappa\left(\frac{\Phi(t)-\Phi(-t)}{t}\right) .
\end{aligned}
$$

Finally, we impose

$$
\begin{aligned}
\Delta_{\kappa}^{\beta}\left[\frac{\chi}{\varphi}\right](t) \\
=v_{1}(\beta, t)\left[\frac{\chi}{\varphi}\right](t)+v_{0}(\beta, t)\left[\frac{\varphi \chi^{\prime}-\chi \varphi^{\prime}}{\varphi^{2}}\right](t)+\kappa\left(\frac{\left[\frac{\chi}{\varphi}\right](t)-\left[\frac{\chi}{\varphi}\right](-t)}{t}\right) \\
=\frac{\nu_{1}(\beta, t)[\varphi \chi](t)+v_{0}(\beta, t)\left[\varphi \chi^{\prime}-\chi \varphi^{\prime}\right](t)}{\varphi^{2}(t)}+\kappa\left(\frac{\left[\frac{\chi}{\varphi}\right](t)-\left[\frac{\chi}{\varphi}\right](-t)}{t}\right) \\
=\frac{\varphi(t)\left[v_{1}(\beta, t) \chi(t)+v_{0}(\beta, t) \chi^{\prime}(t)+\kappa\left(\frac{\chi(t)-\chi(-t)}{t}\right)\right]}{\varphi^{2}(t)} \\
\quad-\frac{\chi(t)\left[v_{1}(\beta, t) \varphi(t)+v_{0}(\beta, t) \varphi^{\prime}(t)+\kappa\left(\frac{\varphi(t)-\varphi(-t)}{t}\right)\right]}{\varphi^{2}(t)}
\end{aligned}
$$




$$
\begin{aligned}
& +\kappa\left(\frac{\left[\frac{\chi}{\varphi}\right](t)-\left[\frac{\chi}{\varphi}\right](-t)}{t}\right)-\frac{\kappa}{\varphi^{2}(t)}\left(\frac{\chi(t)-\chi(-t)}{t}\right)-\frac{\kappa}{\varphi^{2}(t)}\left(\frac{\varphi(t)-\varphi(-t)}{t}\right) \\
& +v_{1}(\beta, t)\left(\frac{\chi}{\varphi}\right)(t) \\
& :=\frac{\varphi(t) \Delta_{\kappa}^{\beta} \chi(t)-\chi(t) \Delta_{\kappa}^{\beta} \varphi(t)}{\varphi^{2}(t)}+\frac{\chi(t)}{\varphi(t)} v_{1}(\beta, t)+K_{\kappa}(t) .
\end{aligned}
$$

Proposition 2.2 The integration by parts of $\Delta_{\kappa}^{\beta}$ satisfies the following integral:

$$
\begin{aligned}
\int_{a}^{b} \varphi(\varsigma) \Delta_{\kappa}^{\beta} \chi(\varsigma) e_{0}(b, \varsigma) d_{\beta} \varsigma= & \left.\varphi(\varsigma) \chi(\varsigma) e_{0}(b, \varsigma)\right|_{a} ^{b}-\int_{a}^{b} \chi(\varsigma)\left[\mathcal{D}^{\beta} \varphi(\varsigma)\right. \\
& \left.-v_{1}(\beta, \varsigma) \varphi(\varsigma)\right] e_{0}(b, \varsigma) d_{\beta} \varsigma+\kappa\langle\varphi, \Lambda\rangle
\end{aligned}
$$

where $\Lambda(\varsigma)=\frac{\chi(\varsigma)-\chi(-\varsigma)}{\varsigma}$.

Proof By the definition of $\Delta_{\kappa}^{\beta}$, we have

$$
\begin{aligned}
& \int_{a}^{b} \varphi(\varsigma) \Delta_{\kappa}^{\beta} \chi(\varsigma) e_{0}(b, \varsigma) d_{\beta} \varsigma \\
& \quad=\int_{a}^{b} \varphi(\varsigma)\left[\mathcal{D}^{\beta} \chi(\varsigma)+\kappa\left(\frac{\chi(\varsigma)-\chi(-\varsigma)}{\varsigma}\right)\right] e_{0}(b, \varsigma) d_{\beta} \varsigma \\
& \quad=\int_{a}^{b} \varphi(\varsigma)\left[\mathcal{D}^{\beta} \chi(\varsigma)\right] e_{0}(b, \varsigma) d_{\beta} \varsigma+\int_{a}^{b} \varphi(\varsigma) \kappa\left(\frac{\chi(\varsigma)-\chi(-\varsigma)}{\varsigma}\right) e_{0}(b, \varsigma) d_{\beta} \varsigma \\
& \quad:=\int_{a}^{b} \varphi(\varsigma)\left[\mathcal{D}^{\beta} \chi(\varsigma)\right] e_{0}(b, \varsigma) d_{\beta} \varsigma+\kappa \int_{a}^{b} \varphi(\varsigma) \Lambda(\varsigma) e_{0}(b, \varsigma) d_{\beta} \varsigma .
\end{aligned}
$$

By using Lemma 1.9 in [11] and the definition of the conformable inner product between two continuous functions, we have the desired conclusion.

In this place, we note that the CDD is not commute operator, because the conformable operator $\mathcal{D}^{\beta}$ is not commute operator [11] for a real case, while it is commute operator in a complex domain [12] for some classes of conformal function (has no zero derivative). Moreover, $\mathrm{CDD}$ is not invariant because the conformable operator $\mathcal{D}^{\beta}$ is not invariant for a real case, while for the set of conformal functions in the open unit disk, the operator $\mathcal{D}^{\beta}$ is invariant [12]. In the next subsection, we shall use the CDD to generalize the definition of the univex functions. These functions are useful in optimization analysis to minimize the EOQ.

\subsection{Generalized univex function}

A class of univex functions is a generalized class of convex functions [17]. This type of functions is usually used to solve multi-objective problems, especially in control theory. Multi-objective difficulties are reflected in control of space constrictions, aeronautical control scheme, and industrial development control, control of production, impulsive control problems and inventory, mechanics, economics, and mechanical engineering difficulties. Here, we aim to employ the CDD to introduce the conformable univex function (CUF). 
Definition 4 For a real-valued function, $\chi$ is a univex function if and only if there are real-valued functions $\Psi, f$, and $g$ satisfying the differential inequality

$$
g(t, \tau) \Psi[\chi(t)-\chi(\tau)] \geq f(t, \tau) \chi^{\prime}(t), \quad g \neq 0 .
$$

Now, by using the conformable operator $\mathcal{D}^{\beta}$ defined in (3), we have the following conformable univex function (CUF).

Definition 5 For a real-valued function, $\chi$ is called a $\beta$-conformable univex function (CUF of order $\beta \in[0,1]$ ) if and only if there are real-valued functions $\Psi, f$, and $g$ satisfying the differential inequality

$$
g(t, \tau) \Psi[\chi(t)-\chi(\tau)] \geq f(t, \tau) \mathcal{D}^{\beta} \chi(t), \quad g \neq 0 .
$$

Consequently, by utilizing the $\operatorname{CDD}\left(\Delta_{\kappa}^{\beta}\right)$, we have the generalized conformable univex function as follows.

Definition 6 For a real-valued function, $\chi$ is called a $(\beta, \kappa)$-conformable univex function of order $\beta \in[0,1]$ and index $\kappa \geq 0$ if and only if there are real-valued functions $\Psi, f$, and $g$ satisfying the differential inequality

$$
g(t, \tau) \Psi[\chi(t)-\chi(\tau)] \geq f(t, \tau) \Delta_{\kappa}^{\beta} \chi(t), \quad g \neq 0 .
$$

\subsection{Economic order quantity}

The pattern of EOQ for the first time was produced by Ford W. Harris [1] as follows:

$$
Q=\sqrt{\frac{2 A B}{C}},
$$

where $A$ indicates the annual demand quantity, $B$ represents the fixed cost for each unit, and $C$ takes the annual holding cost for each unit. In [4], the authors studied the marginal ordinary continuous case taking the form

$$
Q^{\prime}(t)=\Theta(Q(a t), Q(b t), Q(c t))
$$

where $a, b$, and $c$ are nonnegative EOQ model constants indicating to the regular variations in $A, B$, and $C$, while the fractal case of EOQ was studied in [10]. Here, we consider the CDD in Eq. (10) to generalize (16) as follows:

$$
\begin{aligned}
\Delta_{\kappa}^{\beta} Q(t) & =v_{1}(\beta, t) Q(t)+v_{0}(\beta, t) Q^{\prime}(t)+\kappa\left(\frac{Q(t)-Q(-t)}{t}\right) \\
& =v_{1}(\beta, t) Q(t)+v_{0}(\beta, t) \Theta(Q(a t), Q(b t), Q(c t))+\kappa\left(\frac{Q(t)-Q(-t)}{t}\right) .
\end{aligned}
$$

Because of a state in which the consumer uses an EOQ system to control the account of a failing item, when the shortage is not allowed (see [18]), we consider the following 
modification:

$$
\begin{aligned}
\Delta_{\kappa}^{\beta} Q(t)= & v_{1}(\beta, t) Q(t)+v_{0}(\beta, t) \Theta(Q(a t), Q(b t), Q(c t)) \\
& +\kappa\left(\frac{Q(t)-Q(\tau)}{t-\tau}\right), \quad t>\tau .
\end{aligned}
$$

We need to estimate the optimal solution of units according to (17). That is, we minimize the total cost connected with the purchase, distribution, and storing of the manufactured goods. In the existence of a planned customer, who reacts optimally to discount program, the strategy of optimal quantity discount structure by the provider is complex and has to be done sensibly. This is principally happening when the request of the customer is itself unclear. An exciting result called the "reverse bullwhip" takes place where a rise in consumer request uncertainty really decreases order quantity indecision at the provider. There are different methods and theorems to minimize the total cost functions (minimize EOQ) such as the planning horizon theorem [19], stochastic processing [20], and fixed point theorem [4].

From the above information, one can reduce the EOQ model in (18) into the following optimization problem:

$$
\begin{array}{ll}
\text { Minimize } & \Delta_{\kappa}^{\beta} Q(t), \quad t \in J=[0, T] \\
\text { subject to } & Q_{0}=q \leq 0
\end{array}
$$

where $q$ is the initial value of EOQ and $Q$ is continuous for all $t \in J$. For this purpose, we need the following definitions.

Definition 7 For any solution $Q(t)$ for (19), a point $\eta \in \mathfrak{J}:=\{\eta \in J: Q(\eta) \rightarrow 0\}$ is called an efficient point of (19) if there occurs no other point satisfying the inequality $Q(t) \leq Q(\eta)$. Furthermore, it is a weak efficient point if $Q(t)<Q(\eta), \forall t \in J$.

Definition 8 A couple $(\beta, \kappa)-(\chi, \Upsilon)$ is called conformable univex of order $\beta$ if and only if there are real-valued functions $f_{1}, f_{2}, g_{1}$, and $g_{2}$ such that

$$
g_{1}[\chi(t)-\chi(\tau)] \geq f_{1}(t, \tau) \Delta_{\kappa}^{\beta} \chi(t), \quad g_{1} \neq 0,
$$

and

$$
-g_{2}[\Upsilon(t)-\Upsilon(\tau)] \geq f_{2}(t, \tau) \Delta_{\kappa}^{\beta} \Upsilon(t), \quad g_{2} \neq 0
$$

Remark 1 The EOQ model accepts stable demand of a business, product, and immediate availability of items to be re-stocked. It does not account for regular or economic fluctuations. It accepts fixed costs of inventory units, ordering charges, and holding charges. Therefore, this term refers to the contraction in the economy. Consequently, the suggested model is the first model describing this issue by using Dunkl operator.

\section{Results}

In this section, we present some sufficient optimal hypotheses for a point to be an efficient solution of (19) under the conformable $(\beta, \kappa)$-univexity. 
Theorem 3.1 Let $\eta$ be an initial point of the solution of the multi-objective problem (19) and $\epsilon_{1}$ and $\epsilon_{2}$ be two nonnegative constants achieving the following assumptions:

(A) $Q(\eta)=0$ (the initial value of $E O Q$ );

(B) $\epsilon_{1} f_{1}(t, \tau) \Delta_{\kappa}^{\beta} Q(t)+\epsilon_{2} f_{2}(t, \tau) \Delta_{\kappa}^{\beta} q(t)>\epsilon_{1}\left\|g_{1}\right\|+\epsilon_{2}\left\|g_{2}\right\|$;

(C) A couple $(\beta, \kappa)-(\chi, \Upsilon)$ is a conformable univex of order $\beta$;

Then $\eta$ is an efficient solution to minimize (19).

Proof Assume that $\eta$ is not an efficient solution of (19). Therefore, there occurs $x \in \mathfrak{J}$ such that $Q(x) \leq Q(\eta)$. By utilizing assumption $(\mathrm{C})$, we obtain

$$
\epsilon_{1}\left(f_{1}(x, \eta) \Delta_{\kappa}^{\beta} Q(x)\right)<\epsilon_{1}\left\|g_{1}\right\|
$$

and

$$
\epsilon_{2}\left(f_{2}(x, \xi) \Delta_{\kappa}^{\beta} q(x)\right) \leq \epsilon_{2}\left\|g_{2}\right\| .
$$

By taking the summation of (20) and (21), we have

$$
\epsilon_{1}\left(f_{1}(x, \eta) \Delta_{\kappa}^{\beta} Q(x)\right)+\epsilon_{2}\left(f_{2}(x, \eta) \Delta_{\kappa}^{\beta} q(x)\right) \leq \epsilon_{1}\left\|g_{1}\right\|+\epsilon_{2}\left\|g_{2}\right\|,
$$

which contradicts assumption (B). Hence, $\eta$ is an efficient solution which minimizes problem (19). This completes the proof.

Theorem 3.2 Suppose that the following hypotheses are achieved:

(A) $\eta$ is a weakly efficient point of the solution $Q(t)$ of the optimal problem (19);

(B) A couple $(\beta, \kappa)-(Q, q)$ is conformable univex of order $\beta \in[0,1]$ in the direction of $\eta \in \mathfrak{J}$. Moreover, for some $\bar{x} \in \mathfrak{J}$ with $q(\bar{x})<0$,

there occur two constants $\epsilon_{1} \geq 0$ and $\epsilon_{2} \geq 0$ satisfying

$$
\epsilon_{1}\left(f_{1}(x, \eta) \Delta_{\kappa}^{\beta} Q(x)\right)+\epsilon_{2}\left(f_{2}(x, \eta) \Delta_{\kappa}^{\beta} q(x)\right) \geq 0 .
$$

Proof We aim to show that the system

$$
f_{1}(x, \eta) \Delta_{\kappa}^{\beta} Q(x)<0, \quad f_{2}(x, \eta) \Delta_{\kappa}^{\beta} q(x)<0
$$

has no solution for all $x \in \mathfrak{J}$. If the system has at least one solution $y \in \mathfrak{J}$, then in view of Definition 8, we get

$$
Q\left(\eta+\rho_{1} y\right)<Q(\eta) \quad \text { and } \quad q\left(\eta+\rho_{2} y\right)<q(\eta)
$$

for sufficient small arbitrary constants $\rho_{1}, \rho_{2}>0$. By letting $\bar{x}:=\eta+\rho_{2} y$, which leads to $\bar{x} \in \mathfrak{J} \cap N_{\rho_{2}}(\eta)$, in view of assumption (B), one can get

$$
q\left(\eta+\rho_{2} y\right)=q(\bar{x})<0,
$$

which is a contradiction with assumption (A), where $\eta$ is a weak solution $(q(\eta) \geq 0)$. Hence, the above inequalities are nonnegative. Consequently, there exist two nonnegative con- 
stants $\epsilon_{1}$ and $\epsilon_{2}$ achieving the conclusion

$$
\epsilon_{1}\left(f_{1}(x, \eta) \Delta_{\kappa}^{\beta} Q(x)\right)+\epsilon_{2}\left(f_{2}(x, \eta) \Delta_{\kappa}^{\beta} q(x)\right) \geq 0,
$$

with the property $\epsilon_{2} q(\eta) \geq 0$.

This completes the proof.

\section{Remark 2}

- In Theorem 3.2, $\bar{x}$ is called a feasible point, which appears in the solution space. This space represents the initial set of candidate solutions to the optimal problem;

- The optimal problem (19) is a generalization of recent works such as [21-23];

- Minimizing problem (19) is equivalent to minimizing the problem

$$
\begin{array}{ll}
\text { Minimize } & \mathcal{D}^{\beta} Q(t), \quad t \in J=[0, T] \\
\text { subject to } & Q_{0}=q \leq 0
\end{array}
$$

- Theorems 3.1 and 3.2 can be generalized to $\mathbb{R}^{n}$.

\subsection{Numerical examples}

Consider the following data: $\Psi(v)=v, f(t, \tau)=t-\tau, g(t, \tau)=1, \beta=\kappa=0.5$ and $v_{1}(\beta, t)=$ $(1-\beta) t^{\beta}, v_{0}(\beta, t)=\beta t^{1-\beta}, t \in[0,5]$. To minimize problem (19), we have the following calculation:

$$
\Delta_{\kappa}^{\beta} Q(t)=\Delta_{\kappa}^{\beta} \sqrt{t^{3}} \approx t^{4}+0.7 t^{2}+0.7 \sqrt{t}, \quad t \in[0,5],
$$

where $A=B=C=1$. It is clear that $Q(0)=0$, and for arbitrary constants $\epsilon_{1}>0$ and $\epsilon_{2}>0$, we get

$$
f(t, \tau) \Delta_{\kappa}^{\beta} Q(t)=5 * t^{4}+0.7 * t^{2}+0.7 * t^{0.5}>1 .
$$

Thus, in view of Theorem 3.1, the minimization can be recognized when $t \rightarrow 0$ (see Fig. 1).

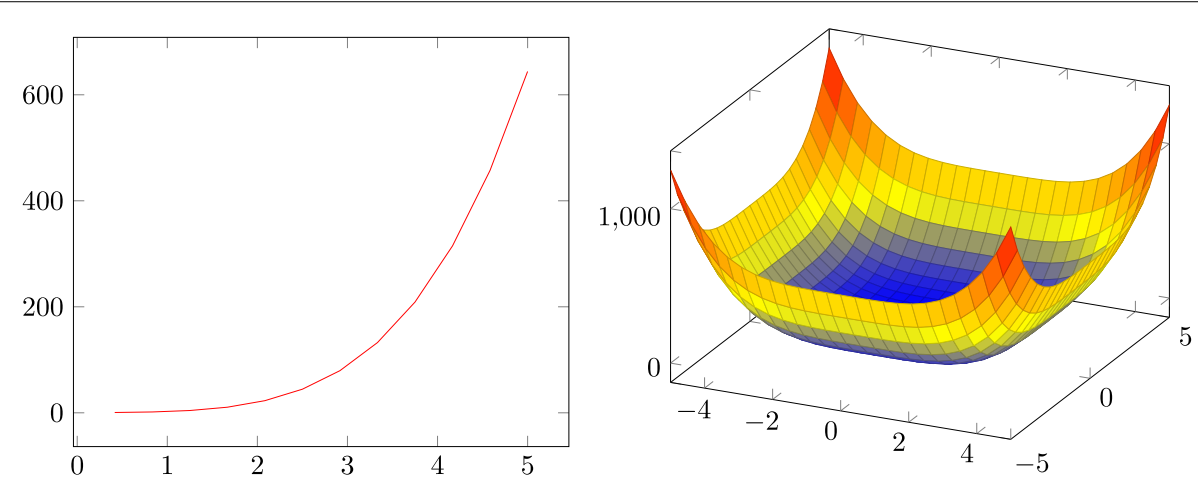

Figure 1 Minimization of 1D and 2D-problem (19) for $f(t, \tau)=t-\tau$. It is clear that minimization appears when $t \rightarrow 0$ 


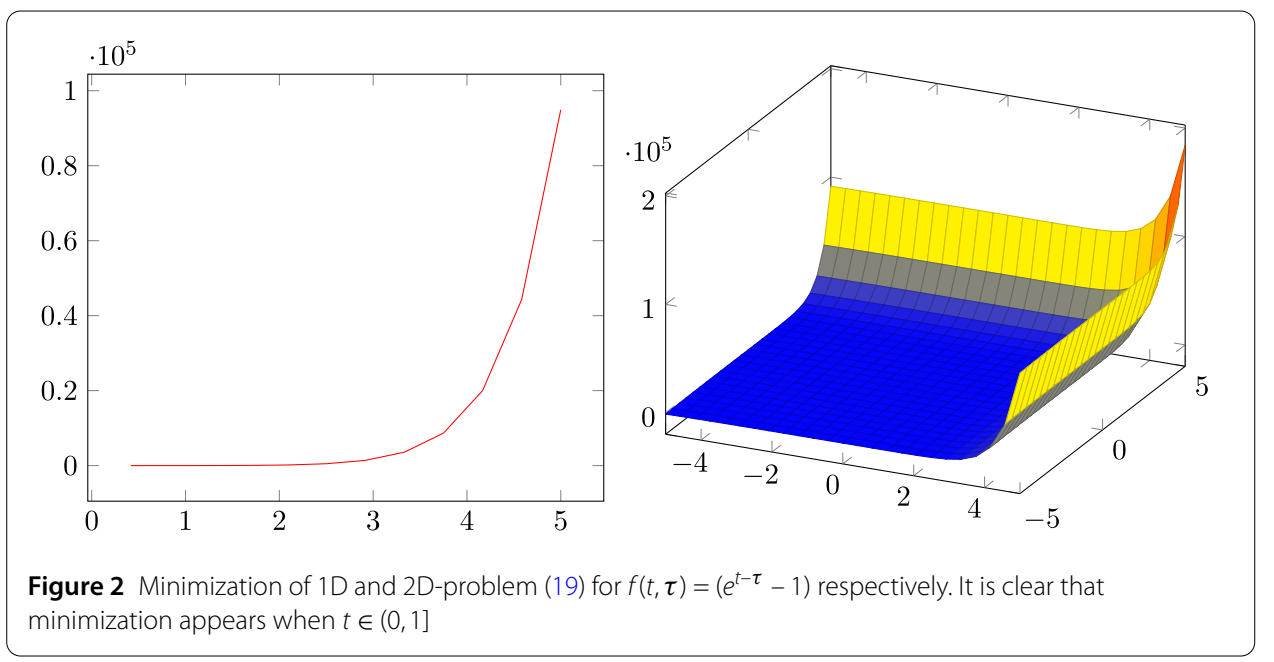

Suppose that $f(t, \tau)=\left(e^{t-\tau}-1\right), t-\tau \geq 1$. Then we obtain

$$
f(t, \tau) \Delta_{\kappa}^{\beta} Q(t)=1.5 * t^{4}+0.7 * t^{2}+0.7 * t^{0.5}>1
$$

Hence, in view of Theorem 3.1, the minimization can be seen in Fig. 2. Similarly, one can optimize problem (19) by using Theorem 3.2.

\section{Acknowledgements}

The authors would like to thank the Ass. Editor for her/his advice to prepare the article. We express our sincere appreciation to the reviewers for their very careful review of our paper and for the comments, corrections, and suggestions that ensued.

\section{Funding}

This work is financially supported by the Prince Sultan University.

\section{Availability of data and materials}

Not applicable.

\section{Competing interests}

The authors declare no conflict of interests.

\section{Authors' contributions}

All authors contributed equally and significantly in writing this article. All authors read and approved the final manuscript.

\section{Author details}

${ }^{1}$ Informetrics Research Group, Ton Duc Thang University, Ho Chi Minh City, Vietnam. ${ }^{2}$ Faculty of Mathematics \& Statistics, Ton Duc Thang University, Ho Chi Minh City, Vietnam. ${ }^{3}$ Deanship of Educational Services, Department of General Sciences, Prince Sultan University, Riyadh, Saudi Arabia. ${ }^{4}$ School of Mathematical and Computer Sciences, Heriot-Watt University, Putrajaya, Malaysia.

\section{Publisher's Note}

Springer Nature remains neutral with regard to jurisdictional claims in published maps and institutional affiliations.

Received: 10 January 2020 Accepted: 28 April 2020 Published online: 12 May 2020

\section{References}

1. Harris, F.W.: How many parts to make at once. Oper. Res. 38(6), 947-950 (1990)

2. Woolsey, G.: A requiem for the EOQ (economic order quantity): an editorial. Hosp. Mater. Manage. Q. 12(1), 82 (1990)

3. Jones, D.J.: JIT \& the EOQ model: odd couple no more! Strateg. Financ. 72(8), 54 (1991)

4. Ibrahim, R.W., Hadid, S.: The EOQ model: a differential cyclic system for calculating economic order quantity. Int. J. Anal. Appl. 16(3), 437-444 (2018)

5. Jaber, M.Y., Nuwayhid, R.Y., Rosen, M.A.: Price-driven economic order systems from a thermodynamic point of view. Int. J. Prod. Res. 42(24), 5167-5184 (2004)

6. Pattnaik, M.: Entropic order quantity (EnOQ) model under cash discounts. Thailand Stat. 9(2), 129-141 (2011) 
7. Pattnaik, M.: Optimization in an instantaneous economic order quantity (EOQ) model incorporated with promotional effort cost, variable ordering cost and units lost due to deterioration. Uncertain Supply Chain Manag. 1(2), 57-66 (2013)

8. Bag, A., Tripathy, P.K., Pattnaik, M.: Entropic order quantity (EnOQ) model for decaying items with partial backordering and lost sale. Int. J. Stat. Syst. 12(4), 803-812 (2017)

9. Sinha, S., Modak, N.M., Sana, S.S.: An entropic order quantity inventory model for quality assessment considering price sensitive demand. Opsearch 57, 88-103 (2020)

10. Ibrahim, R.W., Jafari, H., Jalab, H.A., Hadid, S.B.: Local fractional system for economic order quantity using entropy solution. Adv. Differ. Equ. 2019(1), 96 (2019)

11. Anderson, D.R., Ulness, D.J.: Newly defined conformable derivatives. Adv. Dyn. Syst. Appl. 10(2), 109-137 (2015)

12. Ibrahim, R.W., Jahangiri, J.M., Center, C.C.: Conformable differential operator generalizes the Briot-Bouquet differential equation in a complex domain. AIMS Math. 4(6), 1582 (2019)

13. Dunkl, C.F.: Differential-difference operators associated to reflection groups. Trans. Am. Math. Soc. 311(1), 167-183 (1989)

14. Rosler, M., Voit, M.: Markov processes related with Dunkl operators. Adv. Appl. Math. 21(4), 575-643 (1998)

15. Ibrahim, R.W.: Optimality and duality defined by the concept of tempered fractional univex functions in multi-objective optimization. Int. J. Anal. Appl. 15(1), 75-85 (2017)

16. Ibrahim, R.W., Multi-Objective, D.M.: Optimization using local fractional differential operator. Int. J. Anal. Appl. 17(2), 234-243 (2019)

17. Bector, C.R., Chandra, S., Gupta, S., Suneja, S.K.: Univex sets, functions and univex nonlinear programming. In: Komlosi, S., Rapcsak, T., Schaible, S. (eds.) Proceedings of Conference of Generalized Convexity, Pecs, Hungary, pp. 1-11. Springer, Berlin (1993)

18. Taleizadeh, A.A.: An economic order quantity model for deteriorating item in a purchasing system with multiple prepayments. Appl. Math. Model. 38(23), 5357-5366 (2014)

19. Wagner, H.M., Whitin, T.M.: Dynamic version of the economic lot size model. Manag. Sci. 5(1), 89-96 (1958)

20. Rogers, J.: A computational approach to the economic lot scheduling problem. Manag. Sci. 4(3), 264-291 (1958)

21. Antczak, T., Singh, V: Sufficient optimality conditions and Mond-Weir duality for quasidifferentiable optimization problems with univex functions. Sci. Bull. "Politeh." Univ. Buchar., Ser. A, Appl. Math. Phys. 79(1), 185-196 (2017)

22. Li, L., Zhang, J., Zhou, C.: Optimality conditions for interval-valued univex programming. J. Inequal. Appl. 2019(1), 49 (2019)

23. Antczak, T., Jayswal, A., Jha, S.: The modified objective function method for univex multiobjective variational problems. Bull. Iran. Math. Soc. 45(1), 267-282 (2019)

\section{Submit your manuscript to a SpringerOpen ${ }^{\circ}$ journal and benefit from:}

- Convenient online submission

Rigorous peer review

Open access: articles freely available online

- High visibility within the field

- Retaining the copyright to your article

Submit your next manuscript at $\gg$ springeropen.com 